\title{
Selfish Avoidance Routing Protocol for Mobile Ad Hoc Network
}

\author{
T.V.P.Sundararajan ${ }^{1}$, Dr.A.Shanmugam ${ }^{2}$ \\ ${ }^{1}$ Department of Electronics and Communication Engineering, Bannari Amman Institute \\ of Technology, Sathyamangalam, India
}

tvpszen@yahoo.co.in ${ }^{1}$

Bannari Amman Institute of Technology, Sathyamangalam, India

drasdyahoo.co.in ${ }^{2}$

\begin{abstract}
In an open MANET, different mobile nodes with different goals share their resources in order to ensure global connectivity. However, some resources are consumed quickly as the nodes participate in the network functions. For instance, battery power is considered to be most important in a mobile environment. An individual mobile node may attempt to benefit from other nodes, but refuse to share its own resources. Such nodes are called selfish or misbehaving nodes and their behaviour is termed selfishness or misbehaviour [1]. One of the major sources of energy consumption in the mobile nodes of MANETs is wireless transmission [2]. A selfish node may refuse to forward data packets for other nodes in order to conserve its own energy. Selfish nodes (SN) use the network but do not cooperate, saving battery life for their own communications: they do not intend to directly damage other nodes. This paper proposes Selfish Avoidance Routing Protocol(SARP) for mobile ad hoc network, which takes into account the various factors like the residual battery capacity of a node, power consumption in transmitting packet and the drain rate to increase the battery life of the nodes to overcome the presence of selfish nodes.
\end{abstract}

\section{KEYWORDS}

Cooperation, selfish nodes, ad hoc network, game theory

\section{INTRODUCTION}

Mobile Ad Hoc Network (MANET) is a collection of mobile nodes (hosts) which communicate with each other via wireless links either directly or relying on other nodes as routers. The operation of MANETs does not depend on pre-existing infrastructure or base stations. A mobile node can become a failed node for many reasons, such as moving out of the transmission ranges of its neighbours, exhausting battery power, malfunctioning in software or hardware, or even leaving the network. Besides these failed nodes, based on the behaviour, the mobile nodes are classified into [3], [4],[5]:

- Cooperative Nodes are active in route discovery and packet forwarding, but not in launching attacks

- $\quad$ Failed Nodes are not active in route discovery

- Malicious Nodes are active both in route discovery and launching attacks

Selfish Nodes are active in route discovery, but not in packet forwarding. They tend to drop data packets of others to save their energy so that they could transmit more of their own packets and also to reduce the latency of their packets. This type of attack comes under denial-of-service (DoS) category. 
Selfish nodes, on the other hand, which cooperate during route discovery and defect during packet forwarding, need to be explored. A behavioural model that could dynamically predict the level of cooperation extended by the node towards the network functions such as routing, network monitoring and packet forwarding is therefore, crucial. Selfish nodes, on the other hand, which cooperate during route discovery and defect during packet forwarding, need to be explored. In this paper, we design a behavioural model that could dynamically affect the level of cooperation extended by the node towards the network functions such as routing, network monitoring and packet forwarding.

Ad hoc networks are multi hop networks. In these networks, energy conservation is the key not only extend the life of the nodes but also preventing node become selfish in the network.[6],[7] Enhancing cooperation among nodes is thus, a critical issue in such networks. In principle, energy conservation can be attempted at different layer of networking protocols. This work focuses on the network layer.

Conventional routing algorithms ignore residual battery power of nodes. Links between two nodes are normally established through the shortest path routes. Such algorithm may result in quick depletion of the battery at those nodes that are common to the heavily used routes in the network. Sooner or later the node with depleted battery will be reluctant to participate or withdraw itself in the existing routing which leads to Denial of Service Selfish attack.

Countermeasures against node misbehaviour and selfishness are mandatory requirements in mobile ad hoc networks. [8],[9] Selfishness that causes lack of node activity cannot be solved by classical security means that aim at verifying the correctness and integrity of an operation. In this paper, we propose selfish avoidance routing protocol (SARP) which ensure a node with low residual energy and higher traffic density is not selected for routing. This approach prevents a node become selfish in near future and enhances cooperation among the nodes of a MANET.The effect of power consumption and the current traffic density at a node is also captured with the help of drain rate in our protocol.

The rest of the paper is organized as follows Section 2 presents proposed model of selfish avoidance routing protocol. Sections 3 describes example of operation in resource limited ad hoc network. Section 4 outlines data routing over energy aware path. Scenario study and simulation environment will be discussed in section 6. Performance evaluation of proposed routing protocol with AODV, MMBCR was discussed in Section 7. Finally we conclude our paper.

\section{SELFISH AVOIDANCE ROUTING PROTOCOL (SARP)}

SARP is an on demand routing protocol, where routing decisions are made at each hop. The routes taken by a data packet is based on the cost of routing that packet. The propose cost metric ensures that a node with low residual battery energy and higher traffic density is not selected for routing.

\subsection{Overview of SARP}

The following basic assumptions made in SARP are based on Mobile ad hoc network nodes characteristics and battery characteristics:

- Each node can estimate its own residual power 
- Each node can estimate how much power is consumed in transmitting a packet

- Each node knows its current battery drain rate

- A battery recovers some of its lost capacity when allowed to rest between discharges and in this process; its life is extended due to the charge recovery effect.

\subsection{Route Selection Cost Metric}

All nodes except the destination node calculate their cost, $\mathrm{Ci}$, given by the following equation:

$C i(t)=P\left(\frac{F i}{R i(t)}\right) D R i$

Where, $P$ is the power consumed in transmitting a packet, $F i$ full battery capacity of node $i, D R i$ is the drain rate of node $\mathrm{i}$ which is calculated by Exponential Weighted moving Average Method. $R i(t)$ is the remaining battery capacity of node $i$ at time $t$, and is given by:

$$
R i(t)=R i(t-t l)+\text { Charge recovered in time } t_{1} \ldots
$$

Where $t i$ is is the idle time. This cost metric helps in finding the links with least cost nodes, so that data packets can avoid the nodes with fast depleting batteries. The cost given by this metric is directionally proportional to the power consumption in routing a packet in a node and inversely proportional to the normalized residual battery capacities at these nodes. Drain rate indicates the average energy consumed per unit of time. So nodes with higher traffic densities (i.e higher drain rate) are avoided during routing.

These are the three basic operations in SARP - Route discovery, data Routing and Route maintenance, which are described in the following sub sections.

\subsection{Route Discovery}

SARP is a reactive routing protocol that can construct the route when data transmission is required. In this protocol a source node broadcasts the Route Request packet (RREQ) to the entire network, and all the nodes rebroadcast the received RREQ packet immediately. When a source sends data to a destination whose route is not known, it broadcasts a RREQ along with its coordinates to its neighbours. It also assigns sequence number to this RREQ packet so as to differentiate between old and new sessions. The intermediate nodes that have residual energy greater than threshold $(T c)$, rebroadcast this packet. This threshold $(T c)$ is decreased with the progress of time to ensure that all nodes have their residual battery charge at the same level. The destination, on receiving a RREQ packet, adds that to its route reply list and broadcasts a Route Reply packet (RREP). Intermediate nodes act as a cooperative node and forward these RREP packets towards the source only when their residual energy is greater than threshold $(T c)$ and the following distance condition is satisfied; otherwise the node become selfish nodes and RREP packets are dropped:

$d\left(N_{i}, N_{s}\right) \leq d\left(N_{j}, N_{s}\right)------------(3)$

$d\left(N_{i}, N_{d}\right) \geq d\left(N_{j}, N_{d}\right)$ 
Where $d\left(N_{i}, N_{j}\right)$ is the distance between the pairs of nodes $N i$ and $N j$ and $N i, N j, N s$ and $N d$ are the current, previous hop, source, and destination nodes, respectively.

\subsection{Delay Processing of RREQ}

Many Route Request packets(RREQ) are received at the destination node as in the AODV protocol.[10],[11] The RREQ packet that arrives first is used for the route construction. Moreover, it is not efficient that all the nodes rebroadcast the RREQ packets in the sensor network to reduce the consumed power.

In the proposed protocol, each node is involved in the route construction according to the remaining battery capacity. Each node adjusts the rebroadcast timing of the RREQ packet since the RREQ packet that arrives first is used to construct the route. The rebroadcast timing is determined by the remaining battery capacity. Therefore, when the node receives the RREQ packet from another node, it starts the timer for the rebroadcast of the received RREQ packet by confirming the remaining battery capacity. The timer duration is set to a large value when the battery capacity is small. On the contrary, it is set to a small value when the battery capacity is large. If the rebroadcast of the RREQ packet is delayed, the duration to complete the route construction process becomes large. However, this delay is sufficiently short to convey the detected information to the destination node.

The destination, on receiving a RREQ packet, adds that to its route reply list and broadcasts route Reply packet (RREP), so that all the intermediate can use this information to check the distance condition. Intermediate nodes forward these RREP packets towards the source only when their residual energy is greater than threshold value; otherwise the RREP packets are dropped.

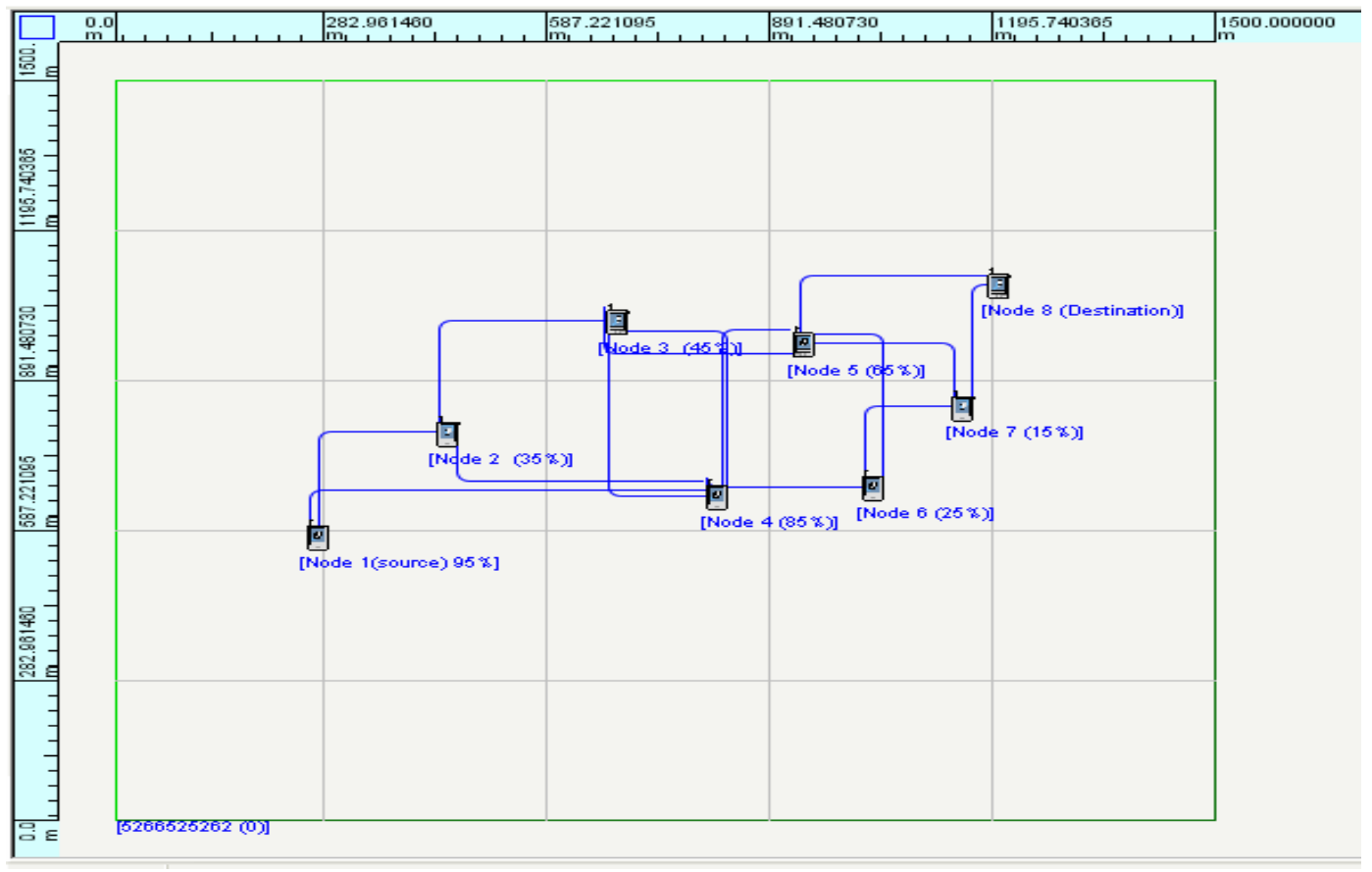

Figure. 1. Route construction of the SARP protocol. 


\subsection{Interruption of RREQ rebroadcast}

Each node immediately rebroadcasts the RREQ packet in the SARP protocol. However, many nodes are positioned in ad hoc networks, and the service areas of each node overlap. Therefore, many RREQ packets are rebroadcast and a considerable amount of the wireless resource is consumed by these packets if each node rebroadcasts them, as in the AODV protocol. Moreover, these redundant rebroadcasts cause the node to waste battery power. In the proposed protocol, until the timer timeout each node interrupts the rebroadcast process of the received RREQ packet when the same RREQ packet is received. Our protocol can prevent the broadcast storm problem of the RREQ packets and reduce the power consumed to rebroadcast redundant RREQ packets.

\section{EXAMPLE OF OPERATIONS}

Figure 1 is an example of ad hoc network, and the operations in the proposed protocol are shown in Fig. 2. In the example, the network comprises seven nodes and one destination node. The value under each node in Fig. 1 indicates the remaining battery capacity. In the proposed protocol, a node that has a large value of the remaining battery capacity actively constructs the route.

The operations are follows.

1) Node1 is a source node initiate a route construction process.

2) Node1 broadcasts a RREQ packet to the network to find a route to a destination node 8 .

3) Nodes 2 and 4 that receive the RREQ packet from node 1 set a timer with a duration that depends on the remaining battery capacity.

4) Node 4 rebroadcasts the RREQ packet in advance since the remaining battery capacity of sensor node 4 is larger than that of node 2 .

5) Node 2 receives the RREQ packet from the node 4 and detects that node 4 retransmits the RREQ packet in advance. Therefore, it stops the timer and interrupts the rebroadcast of the RREQ packet.

6) Nodes 3, 5, and 6 that receive the RREQ packet from node 4 set a timer with the same duration as that of nodes 2 and 4 .

7) Node 5 rebroadcasts the RREQ packet in advance since the remaining battery capacity of node 5 is larger than that of nodes 3 and 6 .

8) Nodes 3 and 6 receive the RREQ packet from the node 5 and detect that sensor node 5 retransmit the RREQ packet in advance. Therefore, they stop the timer and interrupt the rebroadcast of the RREQ packet.

9) The Destination node 8 receives the RREQ packet from node 5 and replies with a route reply (RREP) packet to sensor node 1. Finally, the route via intermediate nodes 4 and 5 is constructed. 


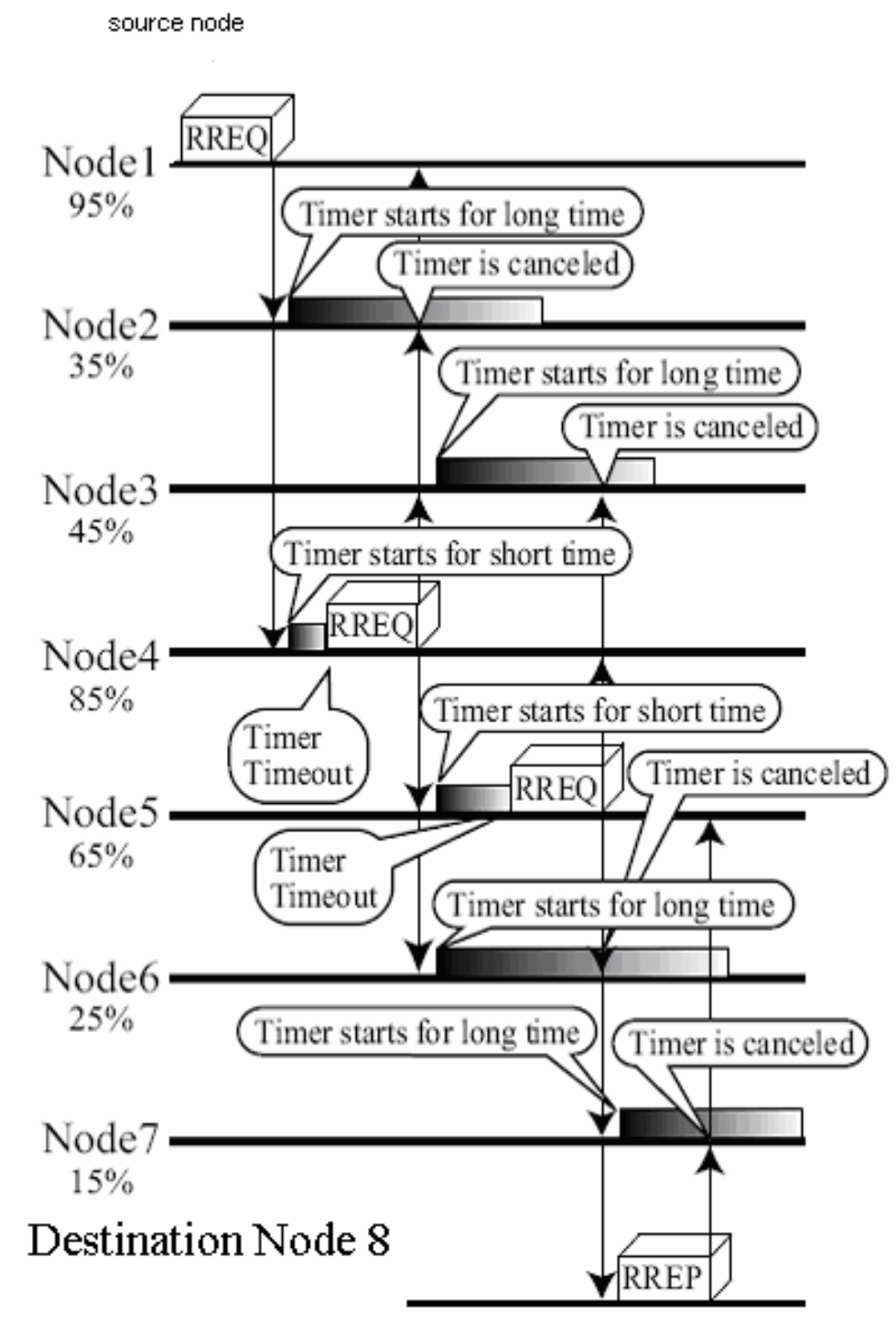

Figure. 2. Operations in the SARP protocol.

\section{DATA ROUTING}

Once a route to the destination is traced, the nodes (including the source) forward the data packets as per their routing tables, selecting the least cost route. When a source node does not require a session any more, it signals for the termination of the session by broadcasting a terminate session (TERM_SESSION) packet. On getting this TERM_SESSION packet, the intermediate nodes with a matching entry in their routing tables delete that entry from their tables. Ultimately, when the destination node receives this packet, it removes this session from its route reply list.

The main improvements of SARP over the other reported routing protocols are: 
- Global information of the energy of all the nodes in the network is not required to be transmitted in SARP. All the nodes on the route are aware of their traffic densities and energy status more accurately. Thus, the nodes with high physical residual energy, already on some routes, are not preferred in route selection.

- Multiple routes are traced during route discovery. They help in providing alternate connectivity between the source and the destination.

- SARP uses a simple intuitive empirical function to capture the charge recovery effect observed in the batteries of mobile nodes, and the values of the various constants for different type of batteries are found out using simple experiments.

- The dynamic variation of the threshold $\left(\mathrm{T}_{\mathrm{c}}\right)$ function ensures that all nodes consume energy, so that they maintain approximately equal energy levels. The node that consumes its energy too fast will be protected from routing, while others will be encouraged. In this way, the energy consumption will be balanced in the whole network.

\section{SIMULATION}

\section{Simulation Environment}

The Qualnet V4.5 network simulator has been used for the simulation studies. Qualnet is a discrete event simulator, which is capable of simulating various kinds of wired/wireless networks at different protocol layers..[12],[13] All the simulations were done at the network layer by using a routing agent plugged on top of the MAC layer agent. The layer above the routing layer (transport level agent or application itself) calls the routing agent when it has to send a packet to a given node in the network. It is the job of the routing agent to determine the next hop for the packet, before it passes the packet down to the MAC layer agent. The MAC layer protocol used for this study was the IEEE 802.11 protocol with User Datagram Protocol (UDP) as the underlying transport agent. All data packets were generated using an application that generates packets at a Constant Bit Rate (CBR). All the nodes used the same packet size of 512 bytes. The power required to transmit and receive a message are a function of the time required for transmitting and receiving a packet are assumed to be:

$$
\begin{aligned}
& P_{t x t}=p_{t x} x t_{t x} \\
& P_{r e c}=p_{r x} x t_{r x}
\end{aligned}
$$

Where $p_{t x}=0.6$ and $p_{r x}=0.3$ (assumption) and $t_{t x}$ and $t_{r x}$ represent the time required to transmit and receive a packet. It can be observed that more the contention, the more will be the time spent in transmitting/receiving the packets and thus, more the power consumed in transmitting the packets.

Each simulation experiment was run for a period of 200 seconds. The links among the nodes were setup and broken at different points of time for varying durations. The connection setup was done randomly for all the scenarios. The mobility model in ns- 2 is based on the "random waypoint model" [14],[15]. In this model, a node travels at a certain speed in a given direction for a certain period of time. After that, its pauses at the destination for a given period of time, and again resumes its journey in another possible direction, and with a different speed. In this simulation study for mobile networks, the speed chosen was between a minimum value of $1 \mathrm{~m} / \mathrm{s}$ and a maximum value of $10 \mathrm{~m} / \mathrm{s}$. The pause time varied according to the degree of mobility required.

\section{PERFORMANCE EVALUATION}


The performance of proposed protocol is evaluated using Qualnet V4.5. Network life, Packet Delivery Ratio, Average End- to-End Delay, Average remaining battery rate, Active cooperative nodes rate are used as metrics to compare the performance of SARP with routing protocol MMBCR,AODV.[16],[17],[18],[19]. Table 2 lists the simulation parameters and environment used.

Table 1. Simulation Parameters

\begin{tabular}{|c|c|}
\hline Simulator & QualNet v 4.5 \\
\hline Simulation time & $50[\mathrm{~h}]$ \\
\hline Number of nodes & 30 nodes, 60 nodes. \\
\hline Area & $200[\mathrm{~m}] \times 200[\mathrm{~m}]$ \\
\hline node placement & Random \\
\hline Node mobility & $\begin{array}{l}\text { static (pause time of } 300 \mathrm{sec} \text { ), } \\
\text { low (pause time of } 100 \mathrm{sec} \text { ), } \\
\text { medium (pause time of } 60 \mathrm{sec} \text { ) } \\
\text { and high (pause time of } 20 \mathrm{sec} \text { ). }\end{array}$ \\
\hline Traffic pattern and load & $\begin{array}{l}\text { CBR traffic / at rates of } 8 \mathrm{kbps} \\
\text { and } 56 \mathrm{kbps}\end{array}$ \\
\hline Data packet size & 512 [byte] \\
\hline Communication system & IEEE 802.11 \\
\hline Bandwidth & $2[\mathrm{Mbps}]$ \\
\hline Communication range & $50[\mathrm{~m}]$ \\
\hline Sensing range & $20[\mathrm{~m}]$ \\
\hline Battery capacity & $500[\mathrm{Ah}]$ \\
\hline Propagation path loss model & free space \\
\hline Wireless environment & AWGN \\
\hline Routing protocol & AODV, Proposed routing \\
\hline
\end{tabular}




\section{Average Remaining Battery Capacity}

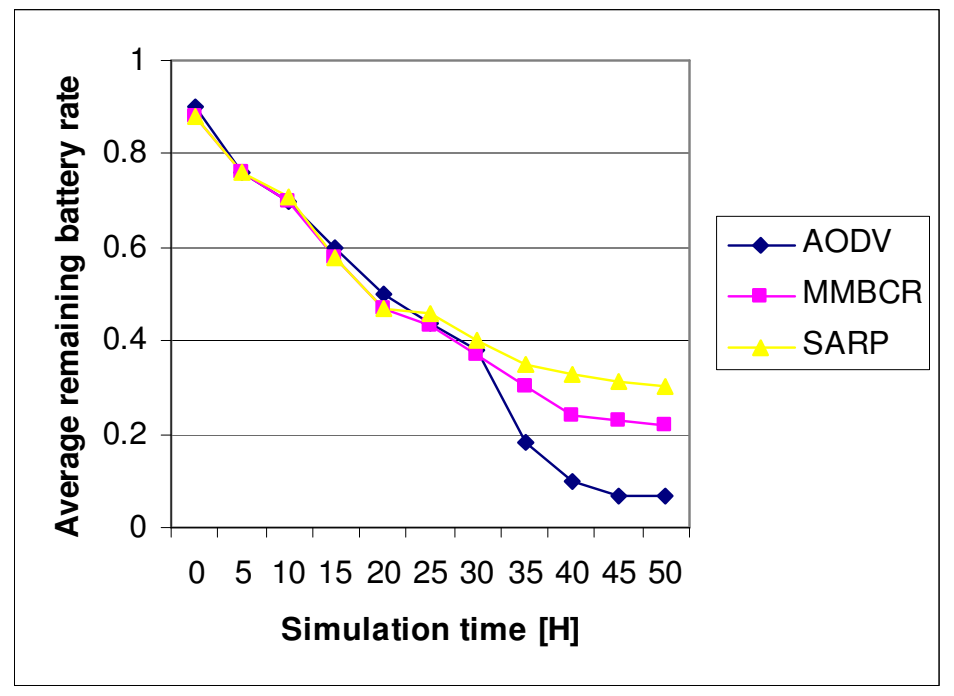

Figure.2 Remaining battery capacity rate

Figure 2 shows the average remaining battery capacity rate of the nodes. From the results, the remaining battery capacity of AODV decreases linearly until 30 [h]. However, it decreases rapidly from $30[\mathrm{~h}]$. This is because the nodes near the source nodes consume a large amount of battery power to forward data packets from a source node which is located far from the destination node. Therefore, the intermediate nodes far from the destination node cannot find the route to the destination node immediately. If the route is not found, each node tries to find it again. As results, many nodes consume a large amount of battery power to find the route to the destination nodes.

On the contrary, the remaining battery capacity of the proposed method (SARP) decreases linearly. Because the intermediate node cancels the retransmission of the RREQ packet, if the intermediate node receives the RREQ packet that it receives. Consequently, a small amount of battery power is consumed if the route delivery process is tried again. Moreover, the remaining battery capacity of the MMBCR protocol also decreases linearly. Because some intermediate nodes with highest battery capacity assist the data packets forwarding to the destination node, and each node can find the route to the destination nodes easily.

\section{Active Cooperative Node Rate}

Figure 3 shows the active cooperative node rate. From the results, the active cooperative node rate of AODV decreases rapidly from $20[\mathrm{~h}]$. This is because the node consumes a large amount of battery power to find the route to the destination node. Therefore, many nodes go down even if the detected events are not arrived at the destination node. 


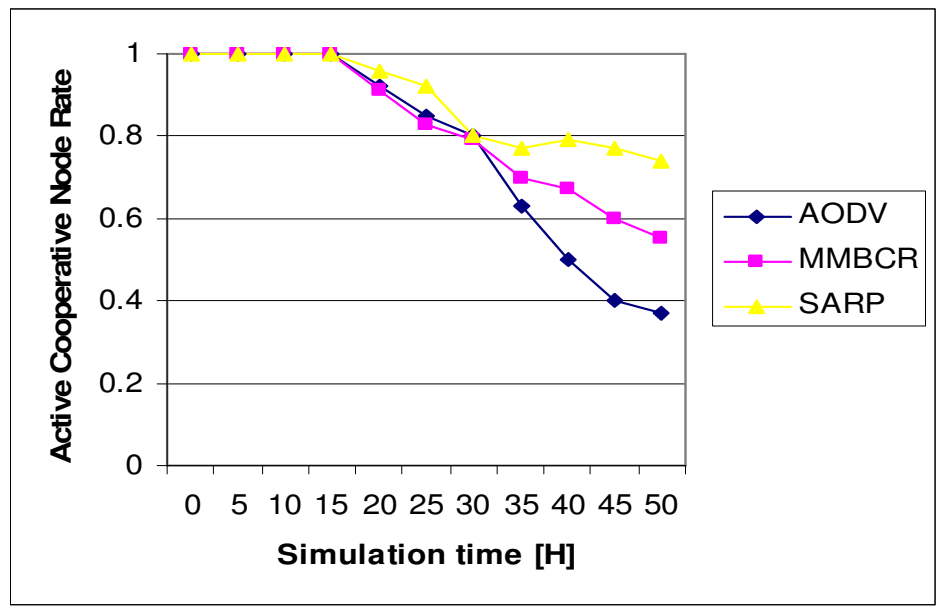

Figure 3 Active Cooperative Node rate

Meanwhile, the active cooperative node rate of the proposed method (SARP) decreases to 80 [\%] at $20[\mathrm{~h}]$ and keeps more than 70 [\%]. Because the proposed method cannot find the route to the destination node from 30 [h]. Therefore, almost intermediate nodes do not consume the battery power a lot. Additionally, the active cooperative node rate of the MMBCR protocol decreases with increasing in the simulation time. This is because the intermediate nodes assist to construct the route to the destination node, almost all nodes can communicate with the destination node.

\section{Number of Route Constructions per Events}

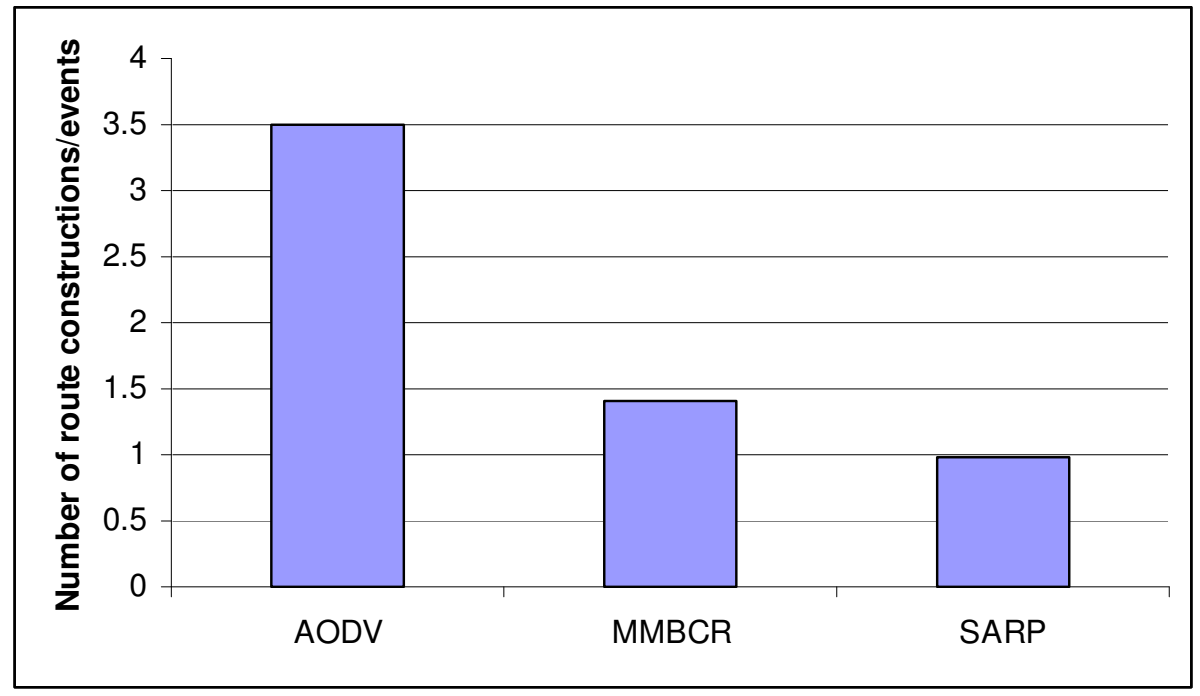

Figure 4. Number of Route Constructions

Figure 4 shows the number of route constructions per events. From the results, we can find that AODV tries to construct the route to the destination node repeatedly. Because AODV may use the intermediate node with a small amount of remaining battery to constructs route. Therefore, several route reconstructions are required. On the contrary, our proposed method (SARP)keeps 
the high active cooperative node rate by considering the remaining battery. As a result, our proposed method can construct the route to the destination node effectively.

\section{Effect of Node Mobility}

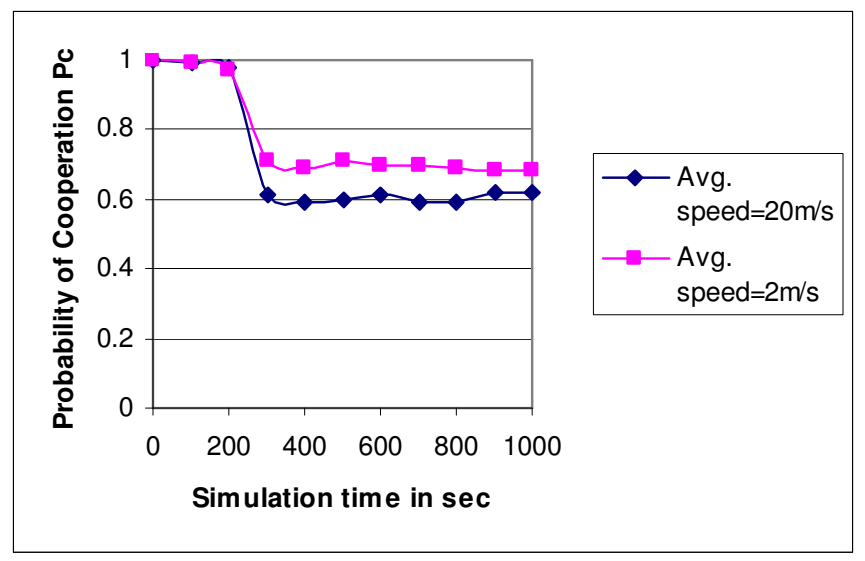

Figure 10 The Effect of Mobility on Probability of Cooperation $P c$

To evaluate the impact of node mobility on $P c$, we conducted simulations using two different average speeds: $20 \mathrm{~ms}$ [Residence time Tin $=350 \mathrm{sec}$ ] and $2 \mathrm{~ms}$ [Residence time Tin $=350 \mathrm{sec}$ ], with 10 movement patterns corresponding to each of them. The simulation results are shown in Figure . 10, in which we can see that the average speed affects Pc considerably, i.e., the higher the mobility is, and the lower $P c$ is. To explain this phenomenon, notice the fact that the faster a node moves, the sooner the node traverses the boundary, yielding a smaller average residence time $T_{\text {in }}$. Consequently, Pc is decreased due to the decreased time spent in the network.

\section{CONCLUSION}

In this chapter, Selfish Avoidance Routing Protocol (SARP) has been developed using a realistic battery model with view to enhance the lifetime of mobile nodes as well as avoid the possibility of node become as selfish nodes. The basic idea of the approach is to keep track of power consumption in routing of packets and recent traffic density at each node, and exploit the charge recovery effect phenomenon observed in batteries. Route selection is based on a cost metric, which captures the residual battery capacity and drain rate of mobile nodes in the network. A low-cost node is one that has high residual battery capacity and low traffic density. The simulation results show that Network life, Average remaining battery rate, Active Cooperative Node rate, Number of Route Constructions are achieved in order to prolong cooperativeness of nodes. However, taking routing decision at each hop may cause some additional delay in routing.

\section{REFERENCES}

[1] H. Miranda and L. Rodrigues, "Preventing Selfishness in Open Mobile Ad Hoc Networks," Proc. Seventh CaberNet Radicals Workshop, Oct. 2002.

[2] L. Buttyan and J.-P. Hubaux, "Security and Cooperation in Wireless Networks," http://secowinet.epfl.ch/, 2006. 
[3] S. Marti, T. Giuli, K. Lai, and M. Baker, "Mitigating Routing Misbehavior in Mobile Ad Hoc Networks," Proc. MobiCom, Aug.2000.

[4] C. E. Perkins, E. M. Royer, and S. Das. RFC 3561: Ad Hoc On Demand Distance Vector (AODV) Routing. http://www.ietf.org/rfc/rfc3561, July 2003.

[5] D. Johnson, D. Maltz, Y.C. Hu, and J. Jetcheva, “The Dynamic Source Routing Protocol for Mobile Ad Hoc Networks (DSR),’Internet draft, Feb. 2002.

[6] L. Buttyan and J.-P. Hubaux, "Enforcing Service Availability in Mobile Ad-Hoc WANs," Proc. MobiHoc, Aug. 2000.

[7] S. Buchegger and J.-Y. Le Boudec, "Performance Analysis of the CONFIDANT Protocol: Cooperation of Nodes, Fairness in Dynamic Ad-Hoc Networks,” Proc. MobiHoc, June 2002.

[8] S. Zhong, J. Chen, and Y.R. Yang, "Sprite: A Simple, Cheat-Proof,Credit-Based System for Mobile Ad-Hoc Networks,” Proc.INFOCOM, Mar.-Apr. 2003.

[9] L. Anderegg and S. Eidenbenz., "Ad hoc-VCG: A truthful and cost efficient routing protocol for mobile ad hoc networks with selfish agents", 9th Annual Intl.Conf. on Mobile Computing and Networking SanDiego, 2003.

[10] J. Crowcroft, R. Gibbens, F. Kelly, and S. Ostring.,"Modelling incentives for collaboration in mobile ad hoc networks", 1st Workshop on Modeling and Optimization in Mobile, Ad Hoc and Wireless Networks", INRIA Sophia-Antipolis, France, 2003.

[11] S. Eidenbenz, G. Resta, and P. Santi, "COMMIT: A sender-centric truthful and energy-efficient routing protocol for ad hoc networks with selfish nodes", IEEE Intl. Parallel and Distributed Processing Symposium- Workshop, Denver, 2005.

[12] URL:http://nesl.ee.ucla.edu/projects/squalnet

[13] Sheeraz Ahmed,Muhammad Bhilal,Umer Farooq, FazleHadi(2007), "Performance Analysis of various routing strategies in Mobile Ad hoc Network using QualNet simulator”, IEEE,1-6,2007.

[14] David oliver jorg(2003)"Performance comparison of MANET Routing Protocols in different environment”, IEEE,1-6,2003

[15] Scalable Network Technologies, "Qualnet simulator",Software Package, 2003[Online]. http://www.scalable-networks.com

[16] Julian Hsu, Sameer Bhatia, Ken Tang, Rajive Bagrodia, Michael J. Acriche, ” Performance of Mobile Ad-Hoc Networking Routing Protocols in Large Scale Scenarios”, pp. 1-7, 2004.

[17] Arun Kumar B.R, Lokanatha C.Reddy, Prakash S.Hiremath, "Performance Comparison of Wireless Mobile Ad-Hoc Network Routing Protocols", IJCSNS International Journal of Computer Science and Network Security, VOL.8 No.6, pp. 337-343, June 2008.

[18] M. Maleki, K. Dantu, M. Pedram, "Lifetime prediction routing in mobile ad hoc networks," IEEE WCNC 2003, pp. 1185 - 1190, Mar. 2003.

[19] J. H. Chang and L. Tassiulas, "Maximum lifetime routing in wireless sensor networks," IEEE/ACM Transactions on Networking, Vol. 12,No. 4, pp. 609 - 619, Aug. 2004. 


\section{Authors}

T.V.P. Sundararajan received the BE Degree in Electronics and Communication from Kongu Engineering College, Perundurai in 1993 and the ME Degree in Applied Electronics from the Government college of technology, coimbatore in 1999. He is Assistant Professor, working in Bannari Amman Institute of Technology, Sathyamangalam. He is doing a part time research in Anna University, Chennai . His current research focuses on mobile ad hoc networks and wireless security. He is member of the IEEE, ISTE and the IEEE computer society.

\section{E-mail : tvpszen@yahoo.co.in}

Dr.A.Shanmugam received the BE Degree in PSG College of Technology in 1972, Coimbatore and ME Degree from College of Engineering, Guindy, Chennai in 1978 and Doctor of Philosophy in Electrical Engineering from Bharathiyar University, Coimbatore in 1994.From 1972-76, he worked as Testing Engineer in Testing and Development Centre, Chennai. He was working as a Lecturer Annamalai University in 1978. He was the Professor and Head of Electronics and Communication Engineering Department at PSG College of Technology,

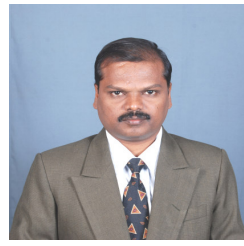
Coimbatore during 1999 to2004. Authored a book titled "Computer

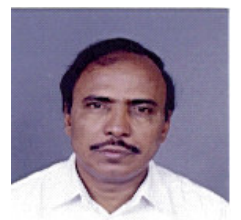
Communication Networks" which is published by ISTE, New Delhi, 2000.He is currently the Principal, Bannari Amman Institute of Technology, Sathyamangalam. .He is on the editorial board of International Journal Artificial Intelligence in Engineering \& Technology (ICAIET), University of Malaysia, International Journal on "Systemics, Cybernetics and Informatics (IJSCI)" Pentagram Research Centre, Hyderabad, India. He is member of the IEEE, the IEEE computer society.

E-mail : dras @ yahoo.co.in 